Research Article,

\title{
The Effect of Kryptonite Bone Cement combined with a Standard Kirschner Wire on Tibial Bone Fracture in Rats
}

\author{
Ibrahim EKE ${ }^{1}$, Mehmet Akif AKCAL ${ }^{2}$, Yusuf IYETIN ${ }^{3}$, Oguz Sükrü POYANLI ${ }^{4}$ \\ ${ }^{1,2}$ MD, Orthopaedics and Traumatology specialist, Orthopaedics and Traumatology Department, Ataturk \\ Public Hospital, Antalya, Turkey \\ ${ }^{3} \mathrm{MD}$, Orthopaedics and Traumatology specialist, Orthopaedics and Traumatology Department, Private \\ Pendik Regional Hospital, Istanbul, Turkey \\ ${ }^{4} \mathrm{MD}$, Orthopaedics and Traumatology specialist, Orthopaedics and Traumatology Department, Medeniyet \\ University School of Medicine, Istanbul, Turkey \\ E-mail Address: dribrahimeke@gmail.com
}

\begin{abstract}
:
Objective

This study aims to observe whether Kryptonite Bone Cement combined with a standard Kirschner wire, instead of autogenous bone grafts, increases bone healing and mechanical strength in rats with tibia fractures.

\section{Methods}

The study included sixteen rats, which were divided into two groups as a control group ( $\mathrm{n}=8)$ and an experimental group $(n=8)$. After segmental fractures were made in both groups, intramedullary fixation of tibia procedures were conducted with the use of a Kirschner wire. No additional procedures were performed in the control group, but Kryptonite Bone Cement was applied in the experimental group. The rats were evaluated clinically, radiologically and histologically 4 times; immediately after the operation, and at the $1^{\text {st }}$, $3^{\text {rd }}$, and $6^{\text {th }}$ weeks following surgery.
\end{abstract}

\section{Results}

One rat from the experimental and one from the control group had both wound dehiscence and wound site infection. No recovery was observed in any rats either in the control or experimental group immediately after surgery. During week 1, callus formation was identified in 5 rats in the experimental group ( $\mathrm{p}=0.0072)$, and during week 3 , fracture lines disappeared in 4 rats in the experimental group $(\mathrm{p}=0.064)$; the differences between control and experimental groups were statistically significant. During week 6, no statistical significance was observed in radiological assessments for the control group and experimental group $(\mathrm{p}=0.71)$.

The rate of non-union was higher in the control group (37.50\%) than in the experimental one, while the rate of complete fusion was higher in the experimental group (87.50\%) than that in the control group.

\section{Conclusion}

Osteoconductivity and ergonomic qualities of Kryptonite Bone Cement prove helpful in bone repair. Future studies to be conducted in a prospective and randomized manner will be effective on demonstrating the effectiveness of Kryptonite Bone Cement.

\section{Introduction:}

What is vital for unhindered healing is the fixation of the fragments sufficiently long enough for osseous closure of the defects. Sufficient cortical bone mass areas require fixation with osteosynthesis plates, screws, or cerclage wires. In 
cases of bone combination, bone fragments cannot be adequately fixed in their orthotopic positions and are left to heal haphazardly [1]. Orthopaedic surgeons seek to ensure vascularisation through periosteal attachments of bone fragments. Although autogenous bone grafts have been known as the "gold standard" for reconstructing a lost bone, there have been various disadvantages such as donor site pain, haematomas, a risk of infection, and nerve injury, which may all lead to significant morbidity. Contrary to such disadvantages, some materials are used to complete cavitary bone defects so that they can provide an adjuvant connected to safe stability of transplanted or reconnected little bone fragments. The optimum material must be resorbable, biocompatible and porous to simplify fast vascularisation and progressive replacement by newly formed tissue [2]. Kryptonite Bone Cement (Doctors Research Group, Inc., Southbury, CT, USA) is a biocompatible polymer made with castor oil. Within 24 hours of continuous application, bone adhesives are shown to bring about rigid bone fixation and stability [3]. The bone cement is composed of isocyanate and polyol with calcium carbonate, associated with exothermic reactions to form an odourless and calcified triglyceride $[4,5]$.

We hypothesized that Kryptonite Bone Cement, when combined with a standard Kirschner wire, would enhance bone healing and mechanical strength. We also believed that it would serve as an adhesive to secure reattached smaller bone fragments and reduce the operation time. In this context, we examined the multiple fragments of a tibial bone fracture in rats histologically and radiologically so as to understand whether or not it could contribute to substantial improvement.

\section{Methods:}

The segmental fracture model was created in rats in the study since fracture recovery is likely to progress with the same biological mechanisms in almost all mammals; the use of a guinea pig, a rat or a mouse model is recommended in studies regarding drug effects or in research conducted on a cellular level $[6,7]$.

The rats were accommodated in separate cages where they could reach on water and food as needed on an occasion at $20^{\circ} \mathrm{C}$ room temperature daily, for 12 hours of light and 12 hours of dark.
Throughout the experiment, the rats were fed with standard rodent feed and tap water, and no animals died until the end of the experiment.

Ethical approval of this study was received from Afyon Kocatepe University Ethics Committee for Test Animals (Afyonkarahisar, Turkey).

\section{Study sample}

Sixteen rats, divided into two groups as the control group $(n=8)$ and the experimental group $(n=8)$, were included in the study. With the segmental fractures in both groups, an intramedullary fixation of the tibia was performed initially with the $0.8-\mathrm{mm}$ Kirschner wire. No additional procedures were performed in the control group, but approximately $6 \mathrm{~mL}$ of adhesive was evenly applied as a thin (1- to 2$\mathrm{mm})$ coating in the experimental group. The formation of bioabsorbable calcified triglyceride (Kryptonite Bone Cement ${ }^{\mathrm{TM}}$ ) involves mixing two liquid components and one powder. A liquid prepolymer isocyanate mixture (methylene diphenyl diisocyanate, $70 \% \mathrm{v} / \mathrm{v}$ ) and an aqueous polyester polyol of polyl (castor oil polyol, 100\% $\mathrm{v} / \mathrm{v})$ were mixed with a dry powder of calcium carbonate $(30 \% \mathrm{w} / \mathrm{w})$. The contents were mixed for 1 minute to 2 minutes with a spatula and applied in a circular motion to bony segments without disturbing their periosteal attachments.

The fracture lines were kept in the convenient anatomical positions for approximately 10 seconds; then the soft tissue and skin were covered with $4 / 0$ silk suture.

\section{Surgical Technique}

Anaesthesia was administered with the intramuscular injection of ketamine $(10 \mathrm{mg} / \mathrm{kg})$. Each rat was shaved in the supine position for surgery on the left lower extremity. Then povidone-soaked, sterile gauze was used to clean up the extremity. The tibia was palpated between the knee joint and the tarsal joint, and the lower extremity was brought to rotation and abduction positions to ensure superior migration of the fibula. The infrapatellar portion of the tibia was palpated so that it would be accessed via the anteromedial incision. The tibia was reached through the cranial tibial muscle, peroneus longus and brevis muscles, and the skin and cutaneous maximus muscular folds were crossed. A $0.8-\mathrm{mm}$ Kirschner wire was advanced to the tarsal joint 
and the tibial tuberosity caudal to the patellar joint was palpated. To create segmental fractures, a 2$\mathrm{mm}$ osteotome was used in the tibial diathesis area starting at nearly $10 \mathrm{~mm}$ caudal to the patellar joint and ending at $10 \mathrm{~mm}$ cranial to the tarsal joint. In this manner, we created four different, equally spaced fragments by means of three osteotomy lines.

\section{Radiological and clinical assessments}

Wound care was done on the surgical site in all rats, which were assessed daily for wound site infection and wound site dehiscence.

Conventional radiological studies were conducted four times after the surgical procedures: immediately after surgery and during weeks 1,3 and 6 . The radiographs were scored numerically according to the scoring system [8].

The rats in both groups were followed for 6 weeks, then they were sacrificed with high doses of ketamine, and the Kirschner wire in the left tibia of each animal was removed. All of the tibias were detached from the surrounding soft tissue without injuring the callus tissue. The fused tissues in tibiae were evaluated independently by two physicians who took part in the study; they used macroscopic and biplanar techniques of examination. The finding of no movement of bone fragments from the fracture line (anteroposterior/lateral) was interpreted as full fusion ( 2 points); malalignment in one plane, as secondary fusion (1 point); and malalignment in both planes, as non-union (0 points) [8].

\section{Histological assessment}

After the ending of the conventional radiological study on week 6 , the tibiae were fixed in $10 \%$ formol solution; the soft tissues were removed the next day, and the tibiae were immersed in $10 \%$ nitric acid. They were kept in those conditions for 2 days and on occasion, controlled by being taken out of the acid. After the completion of the procedure, the tibiae were washed under running water for approximately 1 hour to clean up the acid. Each tibia was longitudinally incised in such a way as to include the site of the lesion and a routine tissue follow-up procedure was achieved. In the final stage, the tissues that were nested in wax were divided into slices of 4 to $5 \mathrm{~mm}$ thick; the slices were stained with haematoxylin and eosin and then examined under a light microscope. The callus tissue was scored according to the system recommended by Huo et al. $[9,10]$.

\section{Statistical analysis}

In this study, the statistical analyses were performed with the NCSS 2007 software package (NCSS, LLC, Kaysville, UT, USA). To assess the data, we used descriptive statistics (median and quartile-deviation) and the Mann-Whitney $U$ test to compare the conditions of the tibiae in the control and experimental groups, as well as chisquare and Fisher's exact tests to compare qualitative data and Mc Nemar's test for the repeated measurements of the qualitative data. To determine the consistency between the two independent observers, the weighted kappa $\left(\kappa_{\mathrm{w}}\right)$ test statistic was used. A p value of less than 0.05 represented significance, and the confidence interval was $95 \%$.

\section{Results:}

\section{Clinical and radiological results}

The rats in the experimental and control groups were evaluated daily, after the surgical procedure for wound site infection and wound site dehiscence. One of the 8 control animals developed both wound site dehiscence and wound site infection. And similarly, one of the 8 experimental animals developed both wound site dehiscence and wound site infection.

After surgery, the experimental group and control group were examined radiologically in the anteroposterior and lateral directions immediately after surgery and during weeks 1,3 and 6 . No recovery was observed in any rats in the control or experimental group immediately after surgery. During week 1, callus formation was identified in 5 rats of experimental group, and the difference between the control and experimental groups was statistically significant $(\mathrm{p}=0.0072)$. During week 3 , the fracture line disappeared in 4 rats in the experimental group, and the difference between control and experimental groups was statistically significant $(\mathrm{p}=0.064)$. During week 6 , no statistically significant difference was observed in the radiological assessments of the control group and experimental group $(\mathrm{p}=0.71)$ (Table 1$)$. 
Ibrahim EKE et. al. /The Effect of Kryptonite Bone Cement combined with a Standard Kirschner Wire on Tibial Bone Fracture in Rats

Table I. Statistical explanation of radiological scoring

\begin{tabular}{|c|c|c|c|c|c|c|}
\hline \multicolumn{2}{|c|}{ Radiologic Scoring } & \multicolumn{2}{|c|}{ Control Group } & \multicolumn{2}{|c|}{ Kryptonite Group } & \\
\hline & & $\mathbf{n}$ & $\%$ & $\mathbf{n}$ & $\%$ & \\
\hline Day 1 & No recovery $(0)$ & 8 & 100 & 8 & 100 & \\
\hline \multirow[t]{2}{*}{ Week 1} & No recovery $(0)$ & 8 & 100 & 3 & 37.50 & \multirow{2}{*}{$\begin{array}{l}X^{2}: 7.27 \\
p=0.007\end{array}$} \\
\hline & Callus formation (1) & $\mathbf{0}$ & 0.00 & 5 & 62.50 & \\
\hline \multirow[t]{3}{*}{ Week 3} & Callus formation (1) & 3 & 37.50 & 1 & 12.50 & \multirow{3}{*}{$\begin{array}{l}X^{2}: 5,50 \\
p=0.064\end{array}$} \\
\hline & Onset of osseous union (2) & 5 & 62.50 & 3 & 37.50 & \\
\hline & $\begin{array}{l}\text { Disappearance of the fracture } \\
\text { line (3) }\end{array}$ & $\mathbf{0}$ & 0.00 & 4 & 50 & \\
\hline \multirow[t]{3}{*}{ Week 6} & Callus formation (1) & 1 & 12.50 & 2 & 25.00 & \multirow{3}{*}{$\begin{array}{l}X^{2}: 0.67 \\
p=0.717\end{array}$} \\
\hline & Onset of osseous union (2) & 2 & 25.00 & 1 & 12.50 & \\
\hline & $\begin{array}{l}\text { Disappearance of the fracture } \\
\text { line (3) }\end{array}$ & 5 & 62.50 & 5 & 62.50 & \\
\hline
\end{tabular}

The manual assessments made by the two independent researchers in both groups before the removal of the tibiae in week 6 were statistically consistent $\left(\kappa_{\mathrm{w}}=0.686, \mathrm{p}=0.0001\right)$. During the manual assessment in the control group, 3 rats demonstrated non-union, while 5 demonstrated complete fusion. In the experimental group, 1 rat demonstrated secondary fusion, and the other 7 demonstrated full fusion (Table II).

A statistical significance was observed between the manual assessment distributions for the control and experimental groups $(\mathrm{p}=0.037)$. The rate of non-union was higher in the control group $(37.50 \%)$ than that in the experimental group, and the rate of complete fusion was higher in the experimental group (87.50\%) than that in the control group (Table II).

Table II. Manual assessment of groups on week 6

\begin{tabular}{|l|l|l|l|l|l|}
\hline \multirow{2}{*}{ Manual Assessment } & \multicolumn{2}{|l|}{ Control Group } & \multicolumn{2}{l|}{ Kryptonite Group } & \multirow{2}{*}{$\begin{array}{l}\mathbf{X}^{2}: 3.82 \\
\mathbf{p}=\mathbf{0 . 1 4 8}\end{array}$} \\
\cline { 2 - 6 } & $\mathbf{n}$ & $\%$ & $\mathrm{n}$ & $\%$ & \\
\hline Non-union & $\mathbf{3}$ & $\mathbf{3 7 . 5 0}$ & $\mathbf{0}$ & $\mathbf{0 . 0 0}$ & \\
\hline Moderate fusion & $\mathbf{0}$ & $\mathbf{0 . 0 0}$ & $\mathbf{1}$ & $\mathbf{1 2 . 5 0}$ & \\
\hline Complete fusion & $\mathbf{5}$ & $\mathbf{6 2 . 5 0}$ & $\mathbf{7}$ & $\mathbf{8 7 . 5 0}$ & \\
\hline
\end{tabular}

\section{Histological results}

During the light microscopic examination with haematoxylin and eosin staining, no foreign body reactions, histotoxic features, inflammation or cortical bone necrosis was seen in the experimental group or the control group. Throughout the examination, the fracture lines were evaluated for the presence of fibrous tissue, cartilage tissue, and immature bone. The data were examined in accordance with the scoring system recommended by Huo et al.[10].

Assessment of the pathological scores of the groups revealed no statistically significant changes $(\mathrm{p}=0.51)$. Recovery with the immature bone was observed both in the control group and in the experimental group (Table III).

Table III. Assessment of pathological scores

\begin{tabular}{|l|l|l|l|l|}
\hline & Control group & Kryptonite group & MW & p* $^{*}$ \\
\hline Pathological scoring & $9(8-9)$ & $8.5(8-9)$ & 26.5 & 0.511 \\
\hline
\end{tabular}

*:MWU Mann-Whitney $U$ test 


\section{Discussion:}

Segmental fractures of the tibia (Müller AO class 42C2) are caused by high-energy trauma. The lesion is characterised by at least two fracture levels (bifocal, trifocal), in which one or several intermediate fracture fragments of tubular bone are separated [1,2]. Even for experienced surgeons, operative treatment of segmental tibia fracture is challenging. Optimal reduction and good stability can be achieved, even in complicated fractures, with plate and screw osteosynthesis [10,11]. In our study, we investigated whether Kryptonite Bone Cement would serve as an adhesive to secure reattached smaller bone fragments, provide mechanical stabilisation of a cortical fragment without interfering with bony union, and reduce the operation time. In clinical practice, such investigations are difficult to perform. However, the primary bones of rats have non-Haversian lamellar structure, and the basic physiological mechanisms of remodelling are similar to those in human bones; this was why we used rats as the experimental animal model for tibial fractures $[1,4]$.

The ideal synthetic material should not conduct heat and should be biocompatible, inert, radio transparent, nonmagnetic, lightweight, rigid, simple to prepare and easily applicable [5]. Kryptonite Bone Cement is close to this ideal except that it is radiopaque and can be moulded. It is also adhesive, bioabsorbable and osteoconductive $[3,5]$. In our study, no foreign body reactions, histotoxic features, inflammation or cortical bone necrosis was observed in the control group or the experimental group. Histological examination verified the ability of Kryptonite Bone Cement to fuse the bone. Kryptonite Bone Cement has osteoconductive properties due to its geometric structure, its low exothermic properties and the presence of calcium carbonate. In fact, in this cement, created by the release of carbon dioxide during the polymerisation process and the addition of calcium carbonate powder, the diameters of the pores ranged from 0.1 to $0.7 \mathrm{~mm}$. This pore size is recognised as ideal for osseous ingrowth $[3,5]$. We also found no significant difference in the pathological scores of the two groups; however, although bone healing was more prominent with immature bone in the control group, the presence of cartilage in addition to immature bone tissue was observed in the experimental group.

In our study, we also investigated the load properties of Kryptonite Bone Cement. Based on the study of Zou et al. and the results of our study, we think that bioabsorbable calcified triglyceride compounds can also be used as an alternative to bone graft in spinal fusion [4]. Di Nuzzo et al. also showed bioabsorbability and osteoconductivity of this cement in six patients who had cranial defects [12]. Kryptonite Bone Cement has been used in cardiothoracic surgery as well, since it enabled rapid sternal fixation and complete elimination of pathological sternal displacement under physiological loading conditions [3].

The limitation of our study was the short followup period; we could not demonstrate the replacement of Kryptonite Bone Cement with bone radiologically and histologically. However, although residual structure of the material remained at the end of the 6-week observation, there was no evidence of inflammation to suggest suppression of bone regeneration.

\section{Conclusion:}

In assessing the mechanical properties of this cement in load-bearing long bones, we found statistically significant differences between the control group and experimental group on week 6 . The non-union rate was higher in the control group $(37.50 \%)$ than in the experimental group, and the rate of complete fusion was higher in the experimental group $(87.50 \%)$ than in the control group. Moreover, we found that with minimal exothermal reaction, Kryptonite Bone Cement did not cause any cortical or soft tissue necrosis in our study. We also observed that this bone cement remained an injectable liquid for up to 10 minutes after mixing, after which it became adhesive. The osteoconductivity and ergonomic qualities of Kryptonite Bone Cement prove helpful in bone repair.

We think that prospective and randomized studies to be conducted in the future will be effective on demonstrating the effectiveness of Kryptonite Bone Cement. 


\section{References:}

[1] Winge, M.I., Reikerås, O. \&Røkkum, M. Arch Orthop Trauma Surg 2011;131: 1035.

[2] Hannouche D, Petite H, Sedel L. Current trends in the enhancement of fracture healing. J Bone Joint Surg Br 2001;83(2):157-64.

[3] Fedak PW, Kolb E, Borsato G, Frohlich DE, Kasatkin A, Narine K, Akkarapaka N, King KM. Kryptonite Bone Cement prevents pathologic sternal displacement. Ann ThoracSurg 2010;90(3):979-85.

[4] Zou J, Zhu X, Shi Q, Wang JC, Yang H. Effect of bioabsorbable calcifiedtriglyceride on spinal fusion in a rodent model. J ClinNeurosci 2010;17(3):360-3.

[5] Uraş I, Yavuz OY, Uygun $M$, Paslioglu E, Kömürcü M. An alternative treatmentmethod for defective pseudoarthrosis; evaluation of eight patients treated withArtelon+Kryptonite.

ActaOrthopBelg 2016;82(1):124-8.

[6] Yilmaz C, Kuyurtar F. Fixation of a talar osteochondral fracture withcyanoacrylate glue. Arthroscopy 2005;21(8):1009.

[7] Alho A, Ekeland A, Strømsøe K, Follerås G, Thoresen BO. Locked intramedullary nailing for displaced tibial shaft fractures. J Bone Joint Surg Br 1990;72(5):805-9.

[8] Akman Ş, Göğüş A, Şener N, Bilgiç B, Aksoy B. The effect of diclofenac sodium administration following rat tibial fractures union: An experimental study.

HaccettepeOrtopediDergisi 2001;11:55-60.

[9] Akcal MA, Poyanli O, Unay K, Esenkaya I, Gokcen B, Firatligil AS. Effect ofN-butyl cyanoacrylate on fracture healing in segmental rat tibia fracture model. J OrthopSurg Res 2014;9:76.

[10] Huo MH, Troiano NW, Pelker RR, Gundberg CM, Friedlaender GE. The influence of ibuprofen on fracture repair: biomechanical, biochemical, histologic, andhistomorphometric parameters in rats. JOrthop Res 1991;9(3):383-90.

[11] Sonderegger J, Grob KR, Kuster MS. Dynamic plate osteosynthesis for fracture stabilization: how to do it. Orthop Rev 2010;2(1):e4.

[12] di Nuzzo G, Luongo M, Parlato C, Moraci A. Cranial reconstruction usingbioabsorbable calcified triglyceride bone cement. J CraniofacSurg 2010;21(4):1170-4. 